J. Austral. Math. Soc. (Series A) 43 (1987), 47-63

\title{
MODULAR INVERSE SEMIGROUPS
}

\section{KATHERINE G. JOHNSTON and PETER R. JONES}

(Received 9 September 1985)

Communicated by T. E. Hall

\begin{abstract}
An inverse semigroup $S$ is said to be modular if its lattice $\mathscr{L} \mathscr{F}(S)$ of inverse subsemigroups is modular. We show that it is sufficient to study simple inverse semigroups which are not groups. Our main theorem states that such a semigroup $S$ is modular if and only if (I) $S$ is combinatorial, (II) its semilattice $E$ of idempotents is "Archimedean" in $S$, (III) its maximum group homomorphic image $G$ is locally cyclic and (IV) the poset of idempotents of each $\mathscr{D}$-class of $S$ is either a chain or contains exactly one pair of incomparable elements, each of which is maximal. Thus in view of earlier results of the second author a simple modular inverse semigroup is "almost" distributive. The bisimple modular inverse semigroups are explicitly constructed. It is remarkable that exactly one of these is nondistributive.
\end{abstract}

1980 Mathematics subject classification (Amer. Math. Soc.): 20 M 10, 08 A 30.

The study of the lattice $\mathscr{L} \mathscr{F}(S)$ of full inverse subsemigroups of an inverse semigroup $S$ was initiated by the latter author in [6], [7]. An inverse semigroup $S$ is called modular if $\mathscr{L} \mathscr{F}(S)$ is modular. (As noted in [6], although modularity of the lattice of all inverse subsemigroups seems a more natural concept to study, the theory is rather trivial.) As a consequence of [6], $S$ is modular if and only if each of its principal factors is, and thus it is sufficient to consider 0 -simple modular inverse semigroups. In fact, as we show, only the simple case need be treated. The following characterization is given (the group and completely 0 -simple cases having been discussed elsewhere).

The first author gratefully acknowledges the support of a research grant from the College of Charleston.

(C) 1987 Australian Mathematical Society 0263-6115/87\$A2.00+0.00 
THEOREM. A simple inverse semigroup $S$ (not a group) with semilattice $E$ of idempotents is modular if and only if

(I) $S$ is combinatorial,

(II) $E$ is Archimedean in $S$ (that is, for any $e, f \in E$ and for any nonidempotent right unit $x$ of $e S e, x^{-n} x^{n} \leqslant f$ for some positive integer $n$ ),

(III) the maximum group homomorphic image of $S$ is locally cyclic, and

(IV) the poset of idempotents of each $\mathscr{D}$-class of $S$ is either a chain or contains exactly one pair of incomparable elements, each of which is maximal.

This theorem is remarkable for its similarity to the description in [7] of distributive simple inverse semigroups: there the second alternative in (IV) cannot occur. Various consequences of the above properties are discussed in Section 4. We briefly mention some of interest here. If $S$ is modular then it is locally distributive (that is, each local submonoid $e S e, e \in E$, is distributive; this usage differs from that current in lattice theory;) its maximum group homomorphic image is also distributive and further the kernel of the least group congruence is distributive and completely semisimple. An important property held by modular inverse semigroups is local $E$-unitariness.

In Section 5 the bisimple modular inverse semigroups are completely described. It is notable that there is precisely one such which is not distributive-the Munn semigroup of the semilattice obtained from an $\omega$-chain, by adjoining two new incomparable maximal elements. The distributive ones are described completely in terms of subgroups of the rationals.

The techniques are in general based on those in [7], but the arguments are rather more difficult. Unfortunately, we do not obtain an analogue of the subdirect decomposition of $\mathscr{L} \mathscr{F}$ found there in the distributive case.

\section{Introduction}

Let $S$ be an inverse semigroup. If $A \subseteq S$ then $E_{A}$ will denote the set of idempotents of $S$ which are in $A ; E_{A}$ is partially ordered in the usual way. We shall generally just write $E$ for $E_{S}$. An inverse subsemigroup of $S$ is termed full if it contains $E$. The full inverse subsemigroups of $S$ form a complete sublattice $\mathscr{L} \mathscr{F}(S)$, or just $\mathscr{L} \mathscr{F}$, of the lattice $(\mathscr{L}(S), \cap, \vee)$ of all inverse subsemigroups. If $S$ is a group then $\mathscr{L} \mathscr{F}=\mathscr{L}$. A significant deviation from the notation of $[6,7]$ is that $\langle A\rangle$ will denote the full inverse subsemigroup generated by the subset $A$ of $S$. For properties of inverse semigroups the reader is referred to $[5,10]$, and for those of lattices to [4]. Recall that a lattice $(L, \wedge, \vee)$ is modular if $a \leqslant b \Rightarrow b \wedge$ $(c \vee a)=(b \wedge c) \vee a$. We shall more often encounter the equivalent form: $L$ is modular if $a \leqslant b \leqslant c \vee a \Rightarrow b=(b \wedge c) \vee a$. 
For completeness we review some of the relevant results from [6].

RESULT 1.1 [6,Theorem 1.4]. For any inverse semigroup $S, \mathscr{L} \mathscr{F}$ is a subdirect product of the lattices of full inverse subsemigroups of its principal factors. Hence $S$ is modular if and only if each principal factor is.

ResUlt 1.2 [6, Corollary 3.6]. A modular completely 0-simple inverse semigroup $S$ is either a modular group with adjoined zero or is a combinatorial Brandt semigroup with at most three nonzero idempotents. In the latter case, if $S$ has fewer than three nonzero idempotents it is in fact distributive.

As mentioned earlier, simple distributive inverse semigroups were characterized in [7] as follows.

Result 1.3. A simple inverse semigroup $S$ is distributive if and only if $S$ satisfies (I)-(III) above and the poset of idempotents of each $\mathscr{D}$-class is a chain.

Modular and distributive groups are discussed in some detail in [11]. Of course every abelian group is modular. The following provides a partial converse which we shall need.

Result 1.4 [11, from Proposition 1.12]. A torsion-free modular group is abelian.

RESULT 1.5 [11, Theorem 1.2]. A group $G$ is distributive if and only if it is locally cyclic.

Thus a torsion-free distributive group is an abelian group of rank 1. It is therefore isomorphic to a subgroup of the additive group $Q$ of rationals [3].

We show next that modularity of inverse semigroups is preserved by quotients. The kernel, $\operatorname{ker} \gamma$, of a congruence $\gamma$ on an inverse semigroup $S$ is the full inverse subsemigroup $\{x \in S: x \gamma e$ for some $e \in E\}$.

Proposition 1.6. Let $\gamma$ be a congruence on an inverse semigroup $S$. The map $\Gamma$ : $\mathscr{L} \mathscr{F}(S) \rightarrow \mathscr{L} \mathscr{F}(S / \gamma)$ induced by $\gamma$ is a join-preserving surjection. When restricted to the principal filter $[\operatorname{ker} \gamma, S], \Gamma$ is a homomorphism upon $\mathscr{L} \mathscr{F}(S / \gamma)$. Hence if $S$ is modular so is $S / \gamma$.

Proof. The first statement is clear. Now let $A, B \in[\mathrm{ker} \gamma, S]$, and suppose $x \in A \Gamma \cap B \Gamma$. Thus $a=a \gamma=b \gamma$ for some $a \in A, b \in B$. Now $a^{-1} b \gamma b^{-1} b$, so $a^{-1} b \in \operatorname{ker} \gamma \subseteq A$. Therefore $a a^{-1} b \in A$, and $a a^{-1} b \in B$ since $a a^{-1} \in B$. So 
$x=\left(a a^{-1} b\right) \gamma \in(A \cap B) \Gamma$. Thus $A \Gamma \cap B \Gamma \subseteq(A \cap B) \Gamma$ and the opposite inclusion is clear.

The following technical lemmas will find repeated use.

LeMma 1.7. Let $S$ be an inverse semigroup and let $A, B \in \mathscr{L F}$. If $x$ is a nonidempotent of $A \vee B$ then $x$ is expressible in the form $x=x_{1} x_{2} \cdots x_{n}$, where each $x_{i}$ is nonidempotent, the $x_{i}$ alternately belong to $A$ and $B$ and $x_{1} \mathscr{R} \times \mathscr{L} x_{n}$. In particular, either $A \cap R_{x x^{-1}}$ or $B \cap R_{x x^{-1}}$ contains the nonidempotent $x_{1}$.

Proof. That each $x_{i}$ may be chosen nonidempotent follows from the fullness of $A$ and $B$. Replacing $x_{1}$ and $x_{n}$ by $x x^{-1} x_{1}$ and $x_{n} x^{-1} x$, respectively, yields $x_{1} \mathscr{R} \times \mathscr{L} x_{n}$.

LEMMA 1.8. Let $S$ be an inverse semigroup and $b \in S, b \notin E$. If $x$ is $a$ nonidempotent in $\langle b\rangle$ then $x=x x^{-1} b^{n}$ for some nonzero integer $n$. Thus $x x^{-1} \leqslant$ $b b^{-1}$ or $x x^{-1} \leqslant b^{-1} b$.

Proof. By [7, Lemma 2.1], $x=e a$ for some $e \in E$, and nonidempotent $a$ in the inverse subsemigroup which $b$ generates: $a=\left(b^{-k} b^{k}\right) b^{n}\left(b^{l} b^{-l}\right)$ for some $k$, $l>0$ and nonzero integer $n$ [10, Chapter IX]. Thus $x=e_{1} b^{n} f_{1}=e_{1}\left(b^{n} f_{1} b^{-n}\right) b^{n}$ for some $e_{1}, f_{1} \in E$, and so $x=x x^{-1} e_{1}\left(b^{n} f_{1} b^{-n}\right) b^{n}=x x^{-1} b^{n}$.

\section{Necessity}

We first show that only simple inverse semigroups need be considered.

Proposition 2.1. Let $S$ be a modular inverse semgroup which is 0-simple but not completely 0-simple. Put $S^{*}=S \backslash\{0\}$. Then $S$ has no zero divisors and $S^{*}$ is a simple inverse semigroup with $\mathscr{L} \mathscr{F}\left(S^{*}\right) \cong \mathscr{L} \mathscr{F}(S)$.

Proof. If $S$ has a zero divisor then there exist nonzero idempotents $e$ and $f$ whose product is 0 ; moreover, by 0 -simplicity $S$ contains a nonzero idempotent $g \mathscr{D} e$ such that $g<f$, so without loss of generality $e \mathscr{D} f$. It is clear that the union $e S e \cup f S f$ is in fact 0-disjoint, whence an inverse subsemigroup of $S$.

Put $A=E \cup e S e, B=E \cup e S e \cup f S f$ and $C=\langle x\rangle$ where $x \in R_{e} \cap L_{f}$. (Recall from $\S 1$ that $\langle x\rangle$ denotes the full inverse subsemigroup generated by $x$.). It is easily verified that $x^{-1}(e S e) x=f S f$, so $B \subseteq C \vee A$. By modularity it follows that $B=(B \cap C) \vee A$. 
Now suppose $y \in(B \cap C) \backslash E$. By Lemma 1.8, $y=y y^{-1} x^{n}$ for some nonzero integer $n$. If $n>0$ then $y y^{-1} \leqslant x x^{-1}=e$ and $y^{-1} y \leqslant x^{-1} x=f$, that is, $y=e y f$. Similarly, if $n<0, g=f y e$. But $y$ also belongs to $B \backslash E \subseteq e S e \cup f S f$, yielding $y=0$ since $e f=0$. Thus $B \cap C \subseteq E$ and $B=A$, a contradiction.

Thus $S^{*}$ is a (simple) inverse subsemigroup of $S$. The map $A \rightarrow A \cup\{0\}$ is easily verified to be an isomorphism of $\mathscr{L} \mathscr{F}\left(S^{*}\right)$ upon $\mathscr{L} \mathscr{F}(S)$.

In the remainder of this section when $S$ is assumed simple it will be implicit that $S$ is not completely simple, that is, not a group. In order to prove (I)-(IV) of the Theorem, several additional properties of modular inverse semigroups will be established. We begin, however, with a direct proof of (I).

Proposition 2.2. A simple modular inverse semigroup is combinatorial.

Proof. Suppose $S$ is not combinatorial. Let $e \in E$ and $c \in H_{e}, c \neq e$. By Simplicity there exists $f \in E, f<e, f \mathscr{D} e$. Let $a \in R_{e} \cap L_{f}$. Now $c=e c e=$ $a b a^{-1}$, where $b=a^{-1} c a \in H_{f}$. Thus $\langle b\rangle \subseteq\langle b, c\rangle \subseteq\langle a\rangle \vee\langle b\rangle$ whence by modularity $\langle b, c\rangle=(\langle b, c\rangle \cap\langle a\rangle) \vee\langle b\rangle$.

Now $c \in\langle b, c\rangle$, so applying Lemma 1.7 there is a nonidempotent $u$ in either $(\langle b, c\rangle \cap\langle a\rangle) \cap R_{e}$ or $\langle b\rangle \cap R_{e}$. However, since $b \in H_{f}, f<e$, it is clear from Lemma 1.8 that $\langle b\rangle \cap R_{e}=\{e\}$. Thus $u \in\langle b, c\rangle \cap\langle a\rangle$.

From $u \in\langle a\rangle$, Lemma 1.8 gives $u=a^{n}$ for some $n>0$. On the other hand from $u \in\langle b, c\rangle$ another application of Lemma 1.7 yields either $u=c^{k}$ (if $u \in\langle c\rangle$ ) or $u=c^{k} b^{l} y$ for some nonzero integers $k$ and $l$, and $y \in S^{1}$. The former case is impossible because $c \in H_{e}$ and $a^{n} \notin H_{e}$. But so is the latter because it implies that $c^{k} b^{l} \mathscr{R} e$, that is, $c^{k} b^{l} b^{-l} c^{-k}=c^{k} f c^{-k}=e$, or $f=e f=$ $\left(c^{-k} c^{k}\right) f\left(c^{-k} c^{k}\right)=c^{-k} c^{k}=e$.

We turn next to (IV), where we must first prove some weaker properties. Recall that the width of a poset $P$ is the cardinality of the largest antichain in $P$. The notation $e \| f$ in $P$ will mean $e$ and $f$ are incomparable.

An element $x$ is called strictly right regular if $e=x x^{-1}>x^{-1} x$, in other words if $x$ is in the right unit subsemigroup $P_{e}=\left\{a \in R_{e}: a e=a\right\}$ of $e S e$ and $x \notin H_{e}$. Strict left regularity is defined dually. Note that the inverse subsemigroup generated by an element of either type is bicyclic.

Proposition 2.3. Let $D$ be a $\mathscr{D}$-class of the simple modular inverse semigroup $S$. Then

(a) $\left(E_{D}, \leqslant\right)$ has width at most 2 , and

(b) $\left(E_{D}, \leqslant\right)$ is locally a chain, that is, if $e, f, g \in E_{D}$, $e>f$ and $e>g$ then $f$ and $\mathrm{g}$ are comparable. 
Proof. (a) Suppose $e, f, g \in E_{D}$, pairwise incomparable. By simplicity there exists $h \in E_{D}, h \leqslant e f g$; let $a \in R_{e} \cap L_{h}, b \in R_{f} \cap L_{h}$ and $c \in R_{g} \cap L_{h}$. Put $x=a b^{-1} \in R_{e} \cap L_{f}$ and $y=b c^{-1} \in R_{f} \cap L_{g}$.

Now $b=b h=b a^{-1} a=x^{-1} a$, so $y=x^{-1} a c^{-1}$. Thus

$$
\langle a\rangle \subseteq\langle a, y\rangle \subseteq\langle c, x\rangle \vee\langle a\rangle
$$

and by modularity

$$
\langle a, y\rangle=(\langle a, y\rangle \cap\langle c, x\rangle) \vee\langle a\rangle .
$$

Applying Lemma 1.7 to $y$, it follows that there is a nonidempotent $z$, say, in $R_{f}$ : either $z \in\langle c, x\rangle \cap\langle a, y\rangle$ or $z \in\langle a\rangle$. By Lemma 1.8, the latter case is impossible, since $a a^{-1}=e \| f$ and $a^{-1} a=h<f$. Applying a similar argument to $z$, then, as a member of $\langle c, x\rangle, z=u w$ for some $w \in S^{1}$ and nonidempotent $u$ of $\langle x\rangle \cap R_{f}$. (By Lemma 1.8, $\langle c\rangle \cap R_{f}=\{f\}$ ). In fact, since $x x^{-1}=e \| x^{-1} x=f$, $\langle x\rangle \cap R_{f}=\left\{f, x^{-1}\right\}$, so $u=x^{-1}$. If $w \neq 1$ then $w$ begins in $c^{ \pm 1}$, whence $x^{-1} c^{ \pm 1} \mathscr{R} f=x^{-1} x$, which would imply that $x x^{-1} \leqslant c c^{-1}=g$ or $x x^{-1} \leqslant c^{-1} c=h$, each a contradiction. Thus $z=x^{-1}$. However a similar argument applied to $z$ as a member of $\langle a, y\rangle$ yields $z=y$. Since $y \neq x^{-1}$ a final contradiction is obtained.

(b) Suppose $e, f, g \in E_{D}, e>f, e>g$ and $f \| g$. Let $b \in R_{g} \cap L_{f}$ and $c \in R_{e}$ $\cap L_{g}$. Put $k=c^{-1} b c \in R_{c^{-1} g c} \cap L_{c^{-1} f c}$. Note that since $f\left\|g, c^{-1} f c\right\| c^{-1} g c$ and also that both $c^{-1} g c$ and $c^{-1} f c$ are below $c^{-1} c=g$. (In particular $g c \in P_{g}$.)

Now since $g, f\left\langle e, b=e b e=c c^{-1} b c c^{-1}=c k c^{-1}\right.$. Thus $\langle k\rangle \subseteq\langle b, k\rangle \subseteq\langle c\rangle$ $\vee\langle k\rangle$ whence, by modularity,

$$
\langle b, k\rangle=(\langle b, k\rangle \cap\langle c\rangle) \vee\langle k\rangle .
$$

By arguments similar to those in (a), $R_{g}$ contains a nonidempotent $u$, say, in $\langle b, k\rangle \cap\langle c\rangle$. If $u \in\langle b\rangle$ then $u=b$, since $b^{2} b^{-2}<g$ and $b^{-1} b \| g$. Similarly if $u \notin\langle b\rangle, u=b k^{i} v$ for some $i \neq 0$ and $v \in S^{1}$ : however this easily yields $f=b^{-1} b$ $\leqslant k^{i} k^{-i} \leqslant k k^{-1}$ or $k^{-1} k$, a contradiction since $k k^{-1}$ and $k^{-1} k$ are below $g$ and $g \| f$. But now $b \in\langle c\rangle$, so $b=g c^{n}=(g c)^{n}$ (since $c g=c$ ) for some $n \neq 0$, which is impossible since $b b^{-1} \| b^{-1} b$ but $g c \in P_{g}$.

Part (b) has the following useful consequence.

Proposition 2.4. Let $S$ be a simple inverse semigroup with the property that for each $\mathscr{D}$-class $D,\left(E_{D}, \leqslant\right)$ is locally a chain. Then for each $e \in E$ the local submonoid eSe has the property that the idempotents of each of its $\mathscr{D}$-classes are totally ordered.

Proof. Let $D$ be a $\mathscr{D}$-class of $e S e$ and suppose $f, g \in E_{D}, f \| g$. (Note that $D$ need not contain $e$, so the hypothesis cannot be directly applied.) There is an idempotent $h$ in $D$ such that $h \leqslant f g$. Let $x \in R_{e} \cap L_{h}$. Then $x^{-1} f x \in E_{D}$ since 
$f \mathscr{R} f x \mathscr{L} x^{-1} f x$, and $x^{-1} g x \in E_{D}$ similarly. But $x^{-1} f x, x^{-1} g x \leqslant x^{-1} x=h \leqslant f$ and $x^{-1} f x \| x^{-1} g x$, contradicting the hypothesis.

We next prove (II) of the Theorem. With the terminology introduced above we may restate " $E$ is Archimedean in $S$ " as "for any $e, f \in E$ and any strictly right regular element $x$ of $R_{e}, x^{-n} x^{n} \leqslant f$ for some positive integer $n$ ".

Proposition 2.5. If $S$ is a simple modular inverse semigroup then $E$ is Archimedean in $S$.

Proof. Let $e, f \in E$ and suppose $x$ is strictly right regular in $R_{e}$, that is, (since $S$ is combinatorial) $x \in P_{e}, x \neq e$. Let $g \in E, g \mathscr{D} e, g<\left(x^{-1} x\right) f$. It is sufficient to prove that $x^{-n} x^{n} \leqslant g$ for some integer $n$. Let $b \in R_{e} \cap L_{g}$. Then $b$ is also in $P_{e}$. Now $x=e x e=b\left(b^{-1} x b\right) b^{-1}$, so $\left\langle b^{-1} x b\right\rangle \subseteq\left\langle x, b^{-1} s b\right\rangle \subseteq\langle b\rangle \vee\left\langle b^{-1} x b\right\rangle$, whnce by modularity, $\left\langle x, b^{-1} x b\right\rangle=\left(\left\langle x, b^{-1} x b\right\rangle \cap\langle b\rangle\right) \vee\left\langle b^{-1} x b\right\rangle$.

Applying Lemma 1.7 to $x$, (and noting that $\left\langle b^{-1} x b\right\rangle \cap R_{e}=\{e\}$ since $b^{-1} x b$ $\in g S g)$, we obtain a nonidempotent $u$ in $\left(\left\langle x, b^{-1} x b\right\rangle \cap\langle b\rangle\right) \cap R_{e}$. Since $u \in$ $\langle b\rangle, u=b^{m}$ for some $m>0$. Now if $u \in\langle x\rangle$ then $u=x^{n}$ for some $n>0$, whence $x^{-n} x^{n}=b^{-m} b^{m} \leqslant b^{-1} b=g$, as required. Otherwise $u=x^{n}\left(b^{-1} x b\right)^{k} w$ for some nonzero integer $k$ and $w \in S^{1}$. In that case, since $b^{-1} x b \in g S g, x^{n} g \mathscr{R} u \mathscr{R} x^{n}$, whence $x^{n} g x^{-n}=x^{n} x^{-n}$ and $x^{-n} x^{n} g=x^{-n} x^{n}$, again as required.

Propositions 2.2 and 2.5 generalize Lemma 2.6 of [7].

As a corollary of the foregoing we obtain several useful subsidiary properties of modular inverse semigroups. We first review some terminology and introduce some notation.

Denote by $\sigma$ the least group congruence on the inverse semigroup $S$. Thus $\sigma=\{(x, y): e x=e y$ for some $e \in E\}$. Throughout, we shall denote the maximum group homomorphic image $S / \sigma$ by $G$. It follows from Proposition 1.6 that if $S$ is modular then so is $G$. A useful observation from [8] is that for any idempotent $e$ of $S$, the natural homomorphism of $S$ upon $G$ maps eSe onto $G$ and induces $\sigma_{e S e}$. Thus $G \cong e S e / \sigma$.

Let $K=\operatorname{ker} \sigma=\{x: e x=e$ for some $e \in E\}$. Then $S$ is $E$-unitary if $K=E$. Equivalently [10, Proposition II.7.2], $S$ is $E$-unitary if $\mathscr{R} \cap \sigma$ is the identical relation on $S$.

The following result was used in [7] to show that distributive inverse semigroups are $E$-unitary.

RESULT 2.6 [7, Lemma 2.7]. Let $S$ be a simple combinatorial inverse semigroup such that $E$ is Archimedean in $S$ and, for each $\mathscr{D}_{\text {-class }} D$ of $S,\left(E_{D}, \leqslant\right)$ is a chain. Then $S$ is E-unitary. 
The following corollary is immediate from Proposition 2.4 (and the easily verified fact that local submonoids inherit simplicity).

COROLlaRY 2.7. Let $S$ be a simple combinatorial inverse semigroup such that $E$ is Archimedean in $S$ and, for each $\mathscr{D}$-class $D$ of $S,\left(E_{D}, \leqslant\right)$ is locally a chain. Then $S$ is locally E-unitary. In particular every simple modular inverse semigroup is locally E-unitary.

Proposition 2.8. Let $S$ be a locally $E$-unitary inverse semigroup. Then $K \backslash E \subseteq$ $\left\{x: x x^{-1} \| x^{-1} x\right\}$.

Proof. By the comments above, for each $e \in E, K \cap e S e=\operatorname{ker} \sigma_{e S e}=E_{e S e}$. If $x x^{-1}$ and $x^{-1} x$ are comparable then $x$ belongs to some $e S e$ and so if $x \notin E$, $x \notin K$.

Eventually it will be shown that for modular inverse semigroups, $K=\{x$ : $\left.x x^{-1} \| x^{-1} x\right\} \cup E$. Next, however, we show how properties of $K$ are reflected in those of $G$.

Proposition 2.9. Let $S$ be a simple modular inverse semigroup. Then $G$ is torsion-free abelian. Thus for each $e \in E$ the right unit subsemigroup $P_{e}$ of eSe is commutative.

Proof. As noted above, $G$ is modular so by Result 1.4 it suffices to prove $G$ is torsion-free.

Let $x \in S$ and suppose $x^{n} \in K$ for some $n>0$, that is, $(x \sigma)^{n}=1$ in $G$. By the previous proposition $x^{n} x^{-n} \| x^{-n} x^{n}$. But then, by considering the known structure of monogenic inverse semigroups, we see that the $n+1 \mathscr{D}$-related idempotents $x^{n} x^{-n},\left(x^{-1} x\right)\left(x^{n-1} x^{-(n-1)}\right), \ldots,\left(x^{-i} x^{i}\right)\left(x^{n-i} x^{-(n-i)}\right), \ldots, x^{-n} x^{n}$ form an antichain (see, for instance [10, Chapter IX]). By Proposition $2.3, n+1 \leqslant 2$, that is, $n=1$ and $x \sigma=1$ in $G$, as required.

The final statement is now immediate from $E$-unitariness of $e S e$.

The following preliminary result, based on [7, Lemma 3.1], will also be used in Section 3 .

LEMMA 2.10. Let $S$ be a combinatorial simple inverse semigroup such that $E$ is Archimedean in $S, G$ is abelian and for each $\mathscr{D}$-class $D,\left(E_{D}, \leqslant\right)$ is locally a chain. Let $A \in \mathscr{L} \mathscr{F}(S)$, let $e \in E$ and suppose $A \cap P_{e} \neq\{e\}$. Then for each $g \in A / \sigma$ there exists $a \in P_{e} \cap A$, ao $=g^{ \pm 1}$. In particular for each $g \in G$ and $e \in E$ there exists $a \in P_{e}, a \sigma=g^{ \pm 1}$. 
Proor. Let $e \in E$. Then the local submonoid $e S e$ satisfies all the above hypotheses and, moreover, by Proposition $2.4\left(E_{D}, \leqslant\right)$ is a chain for each $\mathscr{D}$-class $D$ of $e S e$. Thus eSe satisfies the hypotheses of Lemma 3.1 of [7]. But the consequences of that lemma are precisely those above. The proof is therefore completed by its application to $A \cap e S e$, noting $A / \sigma=e A e / \sigma=(A \cap e S e) / \sigma$ and $P_{e} \subseteq e S e$.

Proposition 2.11. If $S$ is a simple modular inverse semigroup then $G$ is locally cyclic.

Proof. Suppose $G$ has a two-generator subgroup $H$ which is not cyclic. Since $G$ is torsion-free abelian, $H$ therefore has free generators $g$ and $h$, say, with $\langle g\rangle \cap\langle h\rangle=\{1\}$ in $G$.

Let $e \in E$. By the preceding results the above lemma may be applied to $A=S$. Thus there exist $a, b$ in $P_{e}$ with $a \sigma=g, b \sigma=h$ (replacing $g$ or $h$ by its inverse, if necessary). By Proposition 2.9, $a$ and $b$ commute. Further, since $\left(E_{D}, \leqslant\right)$ is locally a chain, without loss of generality $a^{-1} a \leqslant b^{-1} b$.

Then $a=a b b^{-1}=b a b^{-1}=b\left(b^{-1} b a\right) b^{-1}$, so

$$
\left\langle b^{-1} b a\right\rangle \subseteq\langle a\rangle \subseteq\langle b\rangle \vee\left\langle b^{-1} b a\right\rangle,
$$

whence by modularity

$$
\langle a\rangle=(\langle b\rangle \cap\langle a\rangle) \vee\left\langle b^{-1} b a\right\rangle .
$$

Noting that, since $b^{-1} b a \in b^{-1} b S a^{-1} a, R_{e} \cap\left\langle b^{-1} b a\right\rangle=\{e\}$, an application of Lemma 1.7 yields a nonidempotent $u$ in $R_{e} \cap\langle b\rangle \cap\langle a\rangle$. Since $a, b \in P_{e}$, $u=b^{m}=a^{n}$ for some $m, n>0$, that is, $g^{m}=h^{n}$. But since $G$ is torsion-free, this contradicts $\langle g\rangle \cap\langle h\rangle=\{1\}$.

This proves (III) of the Theorem. To complete the proof of necessity it remains to complete that of (IV), by showing that if $x x^{-1}$ and $x^{-1} x$ are incomparable then $x^{-1} x$ (and similarly $\left.x x^{-1}\right)$ is maximal in $\left(E_{D_{x}}, \leqslant\right)$. The first step is to reduce this to the case $x \in K$.

LEMMA 2.12. Under the hypotheses of Lemma 2.10, and thus in any simple modular inverse semigroup, suppose $x x^{-1}$ and $x^{-1} x$ are incomparable but $x \notin K$. Then there exists $y \in K, y \mathscr{D} x$, such that either $y y^{-1}<x x^{-1}$ or $y^{-1} y<x^{-1} x$.

Proof. Let $x \sigma=g \neq 1$, and put $e=x x^{-1}$. By the lemma there exist $a \in P_{e}$ such that $a \sigma=g^{ \pm 1}$. If $a \sigma=g$, put $y=a^{-1} x$. Then $y \in K, y y^{-1}=a^{-1} x x^{-1} a=$ $a^{-1} a<e$ and $y^{-1} y=x^{-1} a a^{-1} x=x^{-1} e x=x^{-1} x$, so $y \mathscr{L} x$. If $a \sigma=g^{-1}$ put $y=a x$. A similar argument yields $y^{-1} y<x^{-1} x$ and $y \mathscr{R} x$.

The proof of (IV) can now be completed. 
Proposition 2.13. Let $S$ be a simple modular inverse semigroup. If $x \in S$ and $x x^{-1} \| x^{-1} x$ then $x^{-1} x$ is maximal in $\left(E_{D_{x}}, \leqslant\right)$.

Proof. By the preceding lemma, it suffices to prove this when $x \in K$. Let $e=x x^{-1}$ and $f=x^{-1} x$ and suppose $f$ is not maximal in $\left(E_{D_{x}}, \leqslant\right)$. Thus $g \mathscr{D} f$, $g>f$ for some $g \in E$. By Proposition $2.3 \mathrm{~b},\left(E_{D_{x^{\prime}}} \leqslant\right)$ is locally a chain, so $g \ngtr e$. Let $a \in R_{e} \cap L_{g}$. Note that $a \notin K$, for otherwise $a^{-1} x \in K$ and $a^{-1} x \in R_{g} \cap L_{f}$, contradicting Proposition 2.8 .

Put $b=a^{-1} x a^{-1}$. Then $b \sigma x^{-2}$, and since $x g=x f g=x f=x$,

$$
b b^{-1}=a^{-1} x g x^{-1} a=a^{-1} x x^{-1} a=g
$$

and

$$
b^{-1} b=a x^{-1} e x a^{-1}=a f a^{-1}=h, \text { say, } h<e .
$$

Now $x=\operatorname{exf}=\operatorname{exg}=a\left(a^{-1} x a^{-1}\right) a=a b a$, so

$$
\langle b\rangle \subseteq\langle b, x\rangle \subseteq\langle a\rangle \vee\langle b\rangle,
$$

whence by modularity

$$
\langle b, x\rangle=(\langle b, x\rangle \cap\langle a\rangle) \vee\langle b\rangle .
$$

Since $b \in g S h, R_{e} \cap\langle b\rangle=\{e\}$, so by Lemma 1.7 there is a nonidempotent $u$ in $R_{e} \cap(\langle b, x\rangle \cap\langle a\rangle)$. In fact since $a a^{-1} \| a^{-1} a, R_{e} \cap\langle a\rangle=\{e, a\}$, so $a=u \in$ $\langle b, x\rangle$ and $a \sigma \in\langle b, x\rangle \sigma=\langle b\rangle \sigma$, since $x \in K$. But this implies $a \sigma b^{n} \sigma a^{2 n}$ for some $n \neq 0$, a contradiction since $G$ is torsion-free.

Combining Propositions 2.3 and 2.13 completes the proof of (IV), and thus of necessity, of the Theorem.

\section{Sufficiency}

Let $S$ be a simple inverse semigroup satisfying (I)-(IV) of the Theorem. From (IV) it follows that for each $\mathscr{D}$-class $D$ of $S,\left(E_{D}, \leqslant\right)$ is locally a chain. In fact if $\left(E_{D}, \leqslant\right)$ is not a chain and $\{e, f\}$ is the unique incomparable pair in $E_{D}$ then the remaining idempotents of $E_{D}$ all lie below $e$ and $f$ and are totally ordered.

Propositron 3.1. The semigroup $S$ is locally E-unitary, $K=\left\{x: x x^{-1} \| x^{-1} x\right\} \cup$ $E$ and for each $e \in E,\left|K \cap R_{e}\right| \leqslant 2$.

Proof. That $S$ is locally $E$-unitary follows from Corollary 2.7, that $K \backslash E \subseteq\{x$ : $\left.x x^{-1} \| x^{-1} x\right\}$ from Proposition 2.8 . 
Suppose $x x^{-1} \| x^{-1} x$ but $x \notin K$. Since the hypotheses of Lemma 2.12 hold there exist incomparable idempotents $y y^{-1}$ and $y^{-1} y$ in $\left(E_{D_{x}}, \leqslant\right)$ such that either $y y^{-1}<x x^{-1}$ or $y^{-1} y<x^{-1} x$, contradicting the maximality of $y y^{-1}$ and $y^{-1} y$. Thus $\left\{x: x x^{-1} \| x^{-1} x\right\} \subseteq K$.

Next let $e \in E$, and suppose $x, y \in\left(K \cap R_{e}\right) \backslash\{e\}$. Then $x x^{-1} \| x^{-1} x$ and $x x^{-1}=y y^{-1} \| y^{-1} y$. By (IV), $x^{-1} x=y^{-1} y$. Since $S$ is combinatorial $x=y$. Thus $K \cap R_{e}=\{e, x\}$.

A preliminary lemma is now required.

LEMMA 3.2. Let $b \in K \backslash E$ and suppose there exist nonidempotents $x \in R_{b}$, $z \in L_{b}$ such that $b \in x S z$. Then $b \in\langle x, z\rangle$.

Proof. Put $e=b b^{-1}, f=b^{-1} b$ and $D=D_{b}$. Since $b \in K, e \| f$ so by (IV), $e$ and $f$ are maximal in $d$ and the remaining idempotents of $D$ form a chain below $e$ and $f$.

Let $b=x y z$ where, without loss of generality, $y y^{-1}=x^{-1} x$ and $y^{-1} y=z z^{-1}$. Suppose $x \in K$. By the proposition $b=x$. Similarly if $z \in K$ then $b=z$. So from now on assume $x, z \notin K$. From the comments above, $x^{-1} x$ and $z z^{-1}$ are comparable and both lie below $e$ and $f$.

Suppose $x^{-1} x=z z^{-1}$. Then $y y^{-1}=y^{-1} y$ and since $S$ is combinatorial $y \in E$. Hence $b \in\langle x, z\rangle$.

Next suppose $x^{-1} x<z z^{-1}=y^{-1} y$. Let $w=\left(y^{-1} y\right) x z$. Then

$$
w w^{-1}=\left(y^{-1} y\right) x z z^{-1} x^{-1}=\left(y^{-1} y\right) x\left(x^{-1} x z z^{-1}\right) x^{-1}=\left(y^{-1} y\right) x x^{-1}=y^{-1} y,
$$

that is, $w \mathscr{R} y^{-1}$. Further, since $b \in K, \quad 1=b^{-1} \sigma=(z \sigma)^{-1}(y \sigma)^{-1}(x \sigma)^{-1}$, so $y^{-1} \sigma x z \sigma w,\left(G\right.$ being abelian). Also $\left(y^{-1}\right)^{-1} y^{-1}=y y^{-1}=x^{-1} x<w w^{-1}$. As above, $w w^{-1}$ and $w^{-1} w$ are comparable; let $g$ be the larger. Then $\left(y^{-1}, w\right) \in \mathscr{R} \cap \sigma$ in the $E$-unitary local submonoid $g S g$, and so $y^{-1}=w$. Therefore $b=x w^{-1} z \in$ $\langle x, z\rangle$.

A similar argument applies if $x^{-1} x>z z^{-1}$.

We now prove modularity of $S$. Suppose $A, B, C \in \mathscr{L F}$ and $A \subseteq B \subseteq C \vee A$. We must show $B \subseteq(B \cap C) \vee A$ (the opposite inclusion being obvious). So let $b \in B \backslash E$. Since $b \in C \vee A$, then by Lemma $1.7, b$ is expressible in the form $b=x_{1} x_{2} \cdots x_{n}$, where the $x_{i}$ are nonidempotents alternately belonging to $C$ and $A, x_{1} \in R_{b}$ and $x_{n} \in L_{b}$.

The case $n=1$ is trivial, so assume $n \geqslant 2$.

Suppose $x_{1} \in A$. Then $b=x_{1}\left(x_{1}^{-1} b\right)$, where $x_{1}^{-1} b \in B$ and $x_{1}^{-1} b=$ $\left(x_{1}^{-1} x_{1} x_{2}\right) \cdots x_{n}$, with $x_{1}^{-1} x_{1} x_{2} \in C$. So it is sufficient to prove $b \in(C \cap B) \vee A$ whenever $x_{1}$ and, similarly, $x_{n}$ belong to $C$. If $b \in K$ then by the lemma $b \in B \cap\left\langle x_{1}, x_{n}\right\rangle \subseteq B \cap C$. So from now on assume $b \notin K$, so that, by Proposition 3.1, $b b^{-1}>b^{-1} b$ without loss of generality. Put $e=b b^{-1}$ : thus $b \in P_{e}$. 
Next suppose $x_{1} \notin K$. (The case $x_{1} \in K$ will be treated later.) By Proposition 3.1, $x_{1}^{-1} x_{1}$ is comparable with $x_{1} x_{1}^{-1}=e$. If $x_{1}^{-1} x_{1}<x_{1} x_{1}^{-1}$ put $c=x_{1}$; if $x_{1}^{-1} x_{1}>x_{1} x_{1}^{-1}$ put $c=x_{1} x_{1}^{-2}$. In either case $c \in C \cap P_{e}$ and $c \notin K$. Let $g=b \sigma$ and $h=c \sigma$. Then since $G$ is locally cyclic and $g, h \neq 1$, there exists $k \in G$ such that $g=k^{m}$ and $h=k^{n}$ for some nonzero integers $m$ and $n$. Applying Lemma 2.10 (with $A=S$ ), there exists $s \in P_{e}$ with $s \sigma=k$ (replacing $k$ by $k^{-1}$, if necessary). Thus $s^{m} \sigma b$ and $s^{n} \sigma c$. If $m$ were negative then $s^{-m}$ would belong to $P_{e}$, as would $s^{-m} b$; but $\left(s^{-m} b\right)^{-1}\left(s^{-m} b\right) \leqslant b^{-1} b<e$, contradicting $s^{-m} b \in K$. Thus $m$ and, similarly, $n$ are positive, whence $s^{m}, s^{n} \in P_{e}$. Since $e S e$ is $E$-unitary, $b=s^{m}$ and $c=s^{n}$. Thus $s^{m n} \in(B \cap C) \cap P_{e}, s^{m n} \neq e$.

To complete the proof we use the following lemma. (Note that Proposition 1.6 proves only that $\Sigma$ is a homomorphism on the interval $[K, S]$ of $\mathscr{L} \mathscr{F}$.)

LEMMA 2.3. On any inverse semigroup $S, \sigma$ induces a homomorphism $\Sigma$ of $\mathscr{L} \mathscr{F}$ upon $\mathscr{L}(G)$.

Proof. As in the proof of Proposition 1.6 it is sufficient to show that $A \Sigma \cap B \Sigma \subseteq(A \cap B) \Sigma$ for $A, B \in \mathscr{L F}$. Let $x \in A \Sigma \cap B \Sigma$. Then $x=a \sigma=b \sigma$ for some $a \in A, b \in B$, whence $e a=e b$ for some $e \in E$. Now $b a^{-1} e a \in B$, since $a^{-1} e a \in E$, and $b a^{-1} e a \in A$ since $b a^{-1} e=b b^{-1} e \in E$. But $\left(b a^{-1} e a\right) \sigma=b \sigma=x$, so $x \in(A \cap B) \Sigma$, as required.

Modularity of $\mathscr{L}(G)$ now yields

$$
B \Sigma=(B \Sigma \cap C \Sigma) \vee A \Sigma=((B \cap C) \vee A) \Sigma .
$$

Thus $g=b \sigma \in((B \cap C) \vee A) \Sigma$. Applying Lemma 2.10 to $(B \cap C) \vee A$ yields an element $t$ of $(B \cap C) \vee A, t \in P_{e}, t \sigma=g^{ \pm 1}$. But $t \sigma \neq g^{-1}$ (otherwise $t b \in K$ and $\left.(t b)^{-1}(t b) \leqslant b^{-1} b<e\right)$. Thus $t \sigma b$ and $E$-unitariness of $e S e$ yields $b=t \in(B$ $\cap C) \vee A$, as required.

The remaining case is where $x_{1} \in K$. Let $y_{1}=x_{1}\left(x_{2} \cdots x_{n}\right)\left(x_{2} \cdots x_{n}\right)^{-1}=$ $b x_{n}^{-1} \cdots x_{2}^{-1}=b x_{n}^{-1} \cdots\left(x_{2}^{-1} y_{1}^{-1} y_{1}\right)$, where $b \mathscr{R} y$ and $x_{2}^{-1} y_{1}^{-1} y_{1} \mathscr{L}_{y_{1}}$. Lemma 3.2 now yields $y_{1} \in\left\langle b, x_{2}^{-1} y_{1}^{-1} y_{1}\right\rangle$. But since $x_{1} \in C, x_{2} \in A$ and so $y_{1} \in B \cap C$. Again, $b=y_{1}\left(y_{1}^{-1} b\right)$ where $y_{1}^{-1} b \in B$ and $y_{1}^{-1} b=\left(y_{1}^{-1} y_{1} x_{2}\right) \cdots x_{n}$. Repeating the above arguments completes the proof of this case.

\section{Properties and examples}

Whilst (I)-(IV) characterize simple modular inverse semigroups, further details of their structure and various alternative descriptions may be elucidated. These illustrate how close to being distributive these semigroups are. 
Proposition 4.1. Let $S$ be a modular inverse semigroup. Then for each $\mathscr{D}$-class $D$ of $S,\left(E_{D}, \leqslant\right)$ is either a chain or a chain with two incomparable maximal elements adjoined.

Proof. This is equivalent to (IV).

Proposition 4.2. A modular inverse semigroup $S$ is locally distributive. In fact $S$ is modular if and only if $S$ is locally distributive and (IV) holds.

Proof. The properties (I)-(III) hold in $S$ if and only if they hold locally. If (IV) also holds then by Proposition 2.4, the $\mathscr{D}$-classes of the local submonoids have totally ordered idempotents. By Result $1.3 S$ is then locally distributive. Conversely, if $S$ is locally distributive then (I)-(III) hold locally, thus in $S$.

We next elucidate properties of $K=\operatorname{ker} \sigma$ and $G=S / \sigma$.

Proposition 4.3. Let $S$ be a simple modular inverse semigroup. Then

(i) $S$ is locally $E$-unitary, and $E$-unitary if and only if distributive;

(ii) $G$ is distributive and is isomorphic with a subgroup of the additive group $Q$ of rationals;

(iii) $K=\left\{x: x x^{-1} \| x^{-1} x\right\} \cup E=\left\{x: x^{3}=x^{2}\right\}$. Thus a nonidempotent of $S$ generates either a bicyclic semigroup or a five element Brandt semigroup;

(iv) $K$ is distributive.

Proof. (i) That $S$ is locally $E$-unitary follows from Corollary 2.7. If $S$ is distributive then $S$ is $E$-unitary (Result 2.6); conversely if $S$ is $E$-unitary then $K=E$. Thus, by (iii) below, $x x^{-1}$ and $x^{-1} x$ are comparable for every $x \in S$, that is, $\left(E_{D}, \leqslant\right)$ is a chain for each $\mathscr{D}$-class $D$, whence $S$ is distributive.

(ii) See Result 1.5 and the comments following it.

(iii) That $K=\left\{x: x x^{-1} \| x^{-1} x\right\} \cup E$ is proven in Proposition 3.1. Clearly if $x^{3}=x^{2}$ then $x \in K$. Conversely, suppose $x \in K \backslash E$, so that $x x^{-1} \| x^{-1} x$. Now $x^{2} \in K$ and $x^{2} \notin E$ then $\left\{x^{2} x^{-2},\left(x x^{-1}\right)\left(x^{-1} x\right), x^{-2} x^{2}\right\}$ forms an antichain in $\left(E_{D_{x}}, \leqslant\right)$, which is impossible by (IV). Thus $x^{2} \in E$, whence $x^{2} \in H_{e}$. Since $S$ is combinatorial, this implies $x^{3}=x^{2}$.

(iv) $K$ is completely semisimple and each of its principal factors contains at most two nonzero idempotents, so distributivity follows from Result 1.2.

We now consider some examples. In view of Proposition 4.1, a semilattice which is bound to play an important role is that in Figure 1, which we shall denote by $Y$. It is obtained from the $\omega$-chain $C_{\omega}=\left\{e_{0}>e_{1}>e_{2} \cdots\right\}$ by adjoining two elements $f_{1}$ and $f_{2}$ covering $e_{0}$. 


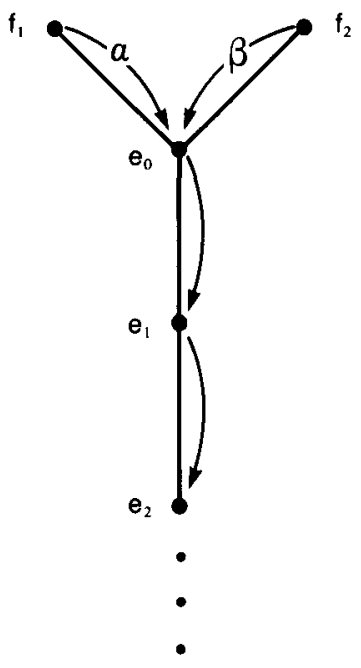

FIGURE 1

Each principal ideal of $Y$ is isomorphic to $C_{\omega}$ so $Y$ is uniform. The Munn semigroup $T_{Y}$ is therefore bisimple. (The reader is referred to [5, Chapter V] for details.) Each local submonoid $e T_{Y} e$ is clearly bicyclic, so $T_{Y}$ is locally distributive. In view of Proposition 4.2, $T_{Y}$ is therefore modular and $T_{Y} / \sigma \cong(Z,+)$. Note that $T_{Y}$ is generated by the two o-related transformations $\alpha: Y f_{1} \rightarrow Y e_{0}$ and $\beta: Y f_{2} \rightarrow Y e_{0}$ shown in Figure 1.

In the next section it will be shown that $T_{Y}$ is the unique modular, nondistributive bisimple inverse semigroup.

In the remainder of this section we consider some examples which are not bisimple. For instance let $A$ be the full inverse subsemigroup of $T_{Y}$ generated by $\alpha^{2}$ and $\beta^{2}$. Since $T_{Y}$ is modular so is $A$. Now $A$ has two $\mathscr{D}$-classes, whose idempotents are $E_{D_{f_{1}}}=\left\{f_{1}, f_{2}, e_{1}, e_{3}, e_{5}, \ldots\right\}$ and $E_{D_{e_{0}}}=\left\{e_{0}, e_{2}, e_{4}, \ldots\right\}$. Thus $S$ is simple but not bisimple. In contrast with the distributive case only the $\mathscr{D}$-class $D_{e_{0}}$ here is a subsemigroup.

That local distributivity does not imply modularity is clear on consideration of the Munn semigroup of the semilattice obtained from $Y$ by adjoining a further covering $f_{3}$ of $e_{0}$.

The strong restrictions placed on the posets of idempotents of $\mathscr{D}$-classes of modular (and distributive) inverse semigroups do not mean that the whole semilattices cannot be quite complicated.

Proposition 4.4. Any semilattice can be embedded in the semilattice of idempotents of some simple distributive inverse semigroup. 
Proof. Let $X$ be a semilattice. We may assume $X=X^{1}$. Form the BruckReilly extension $S=B R(X, \theta)$, where $\theta: X \rightarrow\{1\}$. (See [5, Section V.6] or [10, Section II.5].) Then $S$ is a simple combinatorial inverse monoid. The semilattice $E$ is isomorphic with the ordinal product of $C_{\omega}$ and $X$. (See [10, Corollary II.5.13].) Each element of $X$ corresponds to a unique $\mathscr{D}$-class whose poset of idempotents is isomorphic with $C_{\omega}$. It is easily verified that $E$ is Archimedean in $S$ and $S / \sigma \cong(Z,+)$. Therefore $S$ is distributive.

By replacing the chain $C_{\omega}$ by the semilattice $Y$ in this construction, a class of simple modular nondistributive inverse semigroups can be constructed. We omit the details.

\section{The bisimple case}

The bisimple modular inverse semigroups are completely determined here: in Theorem 5.1 it is shown that $T_{Y}$ (see $\$ 4$ ) is the unique nondistributive instance; in Theorem 5.3 the bisimple distributive inverse semigroups are determined.

Theorem 5.1. Let $Y$ be the semilattice in Figure 1, Section 4. The Munn semigroup $T_{Y}$ is the only modular nondistributive bisimple inverse semigroup.

Proof. Let $S$ be such a semigroup with semilattice $E$. Since $S$ is nondistributive $E$ contains exactly two maximal idempotents, $g_{1}$ and $g_{2}$, say. Put $h_{0}=g_{1} g_{2}$. By Proposition 4.1, $E=\left\{g_{1}, g_{2}\right\} \cup E h_{0}$, and $E h_{0}$ is a chain.

Let $x \in R_{g_{1}} \cap L_{h_{0}}$, and put $h_{i}=x^{-(i+1)} x^{i+1}, i \geqslant 1$. Now $g_{1}$ and $g_{2}$ cover $h_{0}$, and the map $e \rightarrow x^{-1} e x$ is an isomorphism of $E g_{1}$ upon $E h_{0}$, so $x^{-1} x$ covers $x^{-2} x^{2}$. By induction, $x^{-i} x^{i}$ covers $x^{-(i+1)} x^{i+1}$, that is, $h_{i-1}$ covers $h_{i}, i \geqslant 1$. Let $h \in E h_{0}$. Since $E$ is Archimedean in $S$, there is a least positive integer $n$ such that $h_{n-1}=x^{-n} x^{n} \leqslant h$. But $E h_{0}$ is a chain, so $h_{n-2}>h \geqslant h_{n-1}$, whence $h=$ $h_{n-1}$. Hence $E=\left\{g_{1}, g_{2}\right\} \cup\left\{h_{n}: n \geqslant 0\right\}$, clearly isomorphic with $Y$.

Since $S$ is combinatorial it is fundamental, and so is isomorphic to a transitive inverse subsemigroup of $T_{Y}$, [5, Theorem V.6.4]. But $T_{Y}$ itself is combinatorial, so it is its only transitive subsemigroup. Thus $S \cong T_{Y}$.

The bisimple distributive inverse semigroups will be described in terms of lattice-ordered subgroups of $(Q,+)$. We briefly review a construction of Reilly [8,9] generalizing one of Clifford [2].

Let $G$ be an $l$-group written additively, with positive cone $P$. Let $R$ be a filter of $G$ containing $P$, in the sense that if $x \in R$ and $y \geqslant x$ then $y \in R$. Then $(R, P)$ is an $R P$-system in the sense of [8]. Denote by $R^{-1} \circ R$ the set $R \times R$ with 
product $(a, b)(c, d)=\left((c-b)^{+}+a,(b-c)^{+}+d\right)$, (where if $g \in G, g^{+}=g \vee$ $0)$. Then $[8, \S 6], R^{-1} \circ R$ is a bisimple inverse semigroup whose semilattice of idempotents is $E=\{(a, a): a \in R\}$, with $(a, a) \leqslant(b, b)$ if and only if $a \geqslant b$ in $G$. Thus $E$ is order anti-isomorphic with $R$ (under the order induced from $G$ ). Further [8, Theorem 5.3], $\left(R^{-1} \circ R\right) / \sigma \cong G$.

In [9] Reilly called such semigroups l-bisimple, and characterized them abstractly as follows. (For further discussion of the case where $S$ is a monoid, see $[10$, Section X.2].)

ReSUlt 5.2 [9, Theorem 5.4]. A bisimple inverse semigroup $S$ is isomorphic to an l-bisimple inverse semigroup if and only if $S$ is E-unitary, combinatorial and $a P_{e}=P_{e} a$ for all $a \in P_{e}, e \in E$; in that case we may take $G=S / \sigma, P=P_{e}$ and $R=R_{e}$ for some $e \in E$.

Note that if $G=S / \sigma$ is abelian, the last condition is always satisfied, for if $s \in P_{\mathrm{e}}$ then $(a s, s a) \in \mathscr{R} \cap \sigma$, so $a s=s a$ since $S$ is $E$-unitary. As we have noted earlier any simple distributive inverse semigroup $S$ is $E$-unitary and has abelian $G$, so is isomorphic with some $l$-bisimple inverse semigroup. We now make the connection explicit. In the following $(Q,+)$ will be (totally) ordered in the usual way.

THEOREM 5.3. Let $G$ be any nontrivial l-subgroup of $(Q,+)$, with positive cone $P$. Let $R$ be any filter of $G$ containing $P$. Then the semigroup $R^{-1} \circ R$ is a bisimple distributive inverse semigroup. Conversely any bisimple distributive inverse semigroup is isomorphic to an l-bisimple inverse semigroup of this form.

Proof. Let $G, P, R$ be as above and put $S=R^{-1} \circ R$. From the remarks above it follows that $S$ is combinatorial, $S / \sigma \cong G$ is locally cyclic and $E$ is a chain.

To show $E$ is Archimedean in $S$ we use the fact that $(Q,+)$ is an ordered Archimedean group (see [1, Chapter VIII, especially Section 7]), that is, if $g>0$ and $a \in G$ then $n g>a$ for some positive integer $n$. So let $(a, a)$ and $(b, b)$ belong to $E$ and let $(x, y) \in P_{(a, a)},(x, y) \neq(a, a)$. Since $(x, y)(x, y)^{-1}=(x, x)$ and $(x, y)^{-1}(x, y)=(y, y)$ this means $(x, y)=(a, y)$ with $(y, y)<(a, a)$, that is, $y>a$ in $G$. Now $y-a>0$ and so for some positive integer $n, n(y-a)>b$ $-y$, that is, $n(y-a)+y>b$. This is equivalent to the inequality $(a, y)^{-(n+1)}(a, y)^{n+1} \leqslant(b, b)$. Hence $S$ is Archimedean and therefore distributive.

Conversely let $S$ be any bisimple distributive inverse semigroup. As remarked above, $S$ is isomorphic to an $l$-bisimple inverse semigroup $R^{-1} \circ R$, with $G \cong S / \sigma$. Since $G$ is locally cyclic, $G$ is isomorphic with a (nontrivial) subgroup of $(Q,+)$. 
It only remains to show that $G$ is ordered in the usual way. So let $P$ be its positive cone, and suppose $P$ contains $q>0$ (in the natural order). Let $r \in G$, $r \neq 0$. Since $G$ is locally cyclic there exists $g \in G, g>0$ without loss of generality, such that $q=m g$ and $r=n g$, for integers $m$ and $n, m>0$. Now by [1, Theorem XIII.3], since $q \in P$ and $m$ is positive, $g \in P$. By a similar argument, then $r \in P$ if and only if $n>0$, that is $r>0$. So $P=\{r \in G: r \geqslant 0\}$ and $G$ is ordered in the usual way. If, on the other hand, $P$ contains $q<0$, then $P=\{r \in G: r \leqslant 0\}$ and $G$ is ordered in the reverse of the usual way. Then, however, we may replace $G$ by its anti-isomorphic image.

If $S$ is a bisimple distributive inverse monoid then $R$ may be taken equal to $P$. Thus there is a one-one correspondence between such semigroups and nontrivial subgroups of $(Q,+)$. Further, if $G$ is such a subgroup with positive cone $G^{+}$then $R^{-1} \circ R$ is just the inverse hull of $G^{+}$. (See [10, Sections X.1, X.2] for further details.)

\section{References}

[1] G. Birkhoff, Lattice theory (3rd ed., Amer. Math. Soc. Colloq. Publ., Providence, R. I., 1967).

[2] A. H. Clifford, 'A class of $d$-simple semigroups', Amer. J. Math. 15 (1953), 541-556.

[3] L. Fuchs, Infinite abelian groups, Vol. 1 (Academic Press, New York, 1970).

[4] G. Grätzer, General lattice theory (Academic Press, New York, 1978).

[5] J. M. Howie, An introduction to semigroup theory (Academic Press, London, 1976).

[6] P. R. Jones, 'Semimodular inverse semigroups', J. London Math. Soc. 17 (1978), 446-456.

[7] P. R. Jones, 'Distributive inverse semigroups', J. London Math. Soc. 17 (1978), 457-466.

[8] N. R. Reilly, 'Bisimple inverse semigroups', Trans. Amer. Math. Soc. 132 (1968), 101-114.

[9] N. R. Reilly, 'Congruences on a bisimple inverse semigroup in terms of $R P$-systems', Proc. London Math. Soc. (3) 23 (1971), 99-127.

[10] M. Petrich, Inverse semigroups (Wiley, New York, 1984).

[11] M. Suzuki, Structure of a group and the structure of its lattice of subgroups (Springer, Berlin, 1956).

Department of Mathematics

College of Charleston

Charleston, South Carolina 29424

U.S. A.
Department of Mathematics, Statistics and Computer Science Marquette University Milwaukee, Wisconsin 53233 U. S. A. 